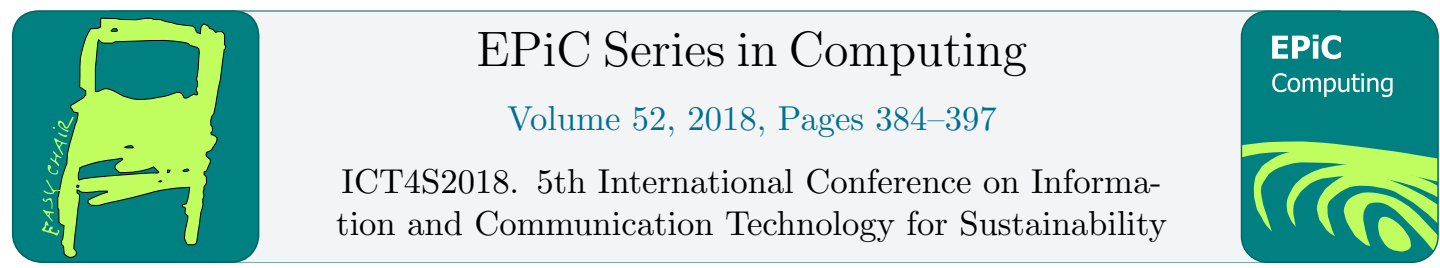

\title{
Undesigning the Internet: An exploratory study of reducing everyday Internet connectivity
}

\author{
Kelly Widdicks ${ }^{1}$, Tina Ringenson ${ }^{2}$, Daniel Pargman ${ }^{3}$, Vishnupriya \\ Kuppusamy $^{4}$, and Patricia Lago ${ }^{5}$ \\ 1 School of Computing and Communications, Lancaster University, UK \\ $\mathrm{k}$.v.widdicks@lancaster.ac.uk \\ 2 School of Architecture and the Built Environment, KTH Royal Institute of Technology, Sweden \\ krin@kth.se \\ ${ }^{3}$ School of Computer Science and Communication, KTH Royal Institute of Technology, Sweden \\ pargman@kth.se \\ 4 Department of Sustainable Communication Networks, University of Bremen, Germany \\ vp@comnets . uni-bremen.de \\ 5 Department of Computer Science, Vrije Universiteit Amsterdam, the Netherlands \\ p.lago@vu.nl
}

\begin{abstract}
Internet connectivity is seamlessly integrated into many of our everyday habits and activities. Despite this, previous research has highlighted that our rather excessive Internet use is not sustainable or even always socially beneficial. In this paper, we carried out an exploratory study on how Internet disconnection affects our everyday lives and whether such disconnection is even possible in today's society. Through daily surveys, we captured what Internet use means for ten participants and how this varies when they are asked to disconnect by default, and re-connect only when their Internet use is deemed as necessary. From our study, we found that our participants could disconnect from the Internet for certain activities (particularly leisure focused), yet they developed adaptations in their lives to address the necessity of their Internet use. We elicit these adaptations into five themes that encompass how the participants did, or did not, use the Internet based on their necessities. Drawing on these five themes, we conclude with ways in which our study can inspire future research surrounding: Internet infrastructure limits; the promotion of slow values; Internet non-use; and the undesign of Internet services.
\end{abstract}

Index Terms - sustainability; everyday life; reduced Internet connectivity; limits; slow values; non-use; undesign.

\section{Introduction}

We live in a time when frequent online presence, hence Internet connectivity, is expected both professionally and privately [39]. Although Internet access differs significantly between countries [14], in many parts of the world it might even be the case that quasi-constant connectivity is

B. Penzenstadler, S. Easterbrook, C. Venters and S.I. Ahmed (eds.), ICT4S2018 (EPiC Series in Computing, vol. 52), pp. 384-397 
expected, at least in some walks of life or for some professions. As a result, we have come to demand more from the online world and from the information and communication technologies (ICT) that enable it [36]. The dominant practice in regards to ICT today is to strive for increasingly faster and richer digital services, where such new services create greater demand; this in turn drives growth of infrastructure capacity, which (again) allows for new services and so on. Preist et al. [25] refer to this reinforcing feedback loop in terms of a 'cornucopian paradigm'. The cornucopian paradigm is unsustainable, since it assumes an ever-growing infrastructure built on finite resources, including rare earth minerals and non-renewable energy resources $[21,25]$. With this as a backdrop, we have formulated a study that explores 'living with less', i.e. assuming less from ICT rather than constantly striving for more (information, speed, connectivity, time spent online).

In designing the experiment, we first considered fully disconnecting from the Internet [18]. While some of us adopt (and enjoy) such a lifestyle occasionally (e.g. on vacations or during leisure time), many of us have occupations that require daily Internet access and also rely on the Internet to sustain important parts of our private lives. Hence, we opted for exploring decreased connectivity, i.e. less access to, or use of, the Internet. This decreased connectivity was facilitated by modes of 'Internet disconnection' and 'Internet connection': turning off Wi-Fi or mobile data on Internet-connected devices by default and turning on for Internet services only when subjectively necessary. Through this decreased connectivity, we were interested in understanding 1) what role the Internet plays in our everyday lives, 2) which parts were more or less necessary for us (or at least significantly harder to refrain from), and 3) which parts would be comparatively easy to reduce or eliminate from our everyday Internet use.

Within our paper, we present five themes for how users did (and did not) adapt to Internet disconnection (ordered in increased necessity): 1) removing Internet use completely; 2) replacing use with other activities; 3) planning for limited use (including pre-disconnection reliability); 4) rationing, postponing and batching Internet use; and 5) abandoning disconnection. We then use these findings to suggest directions for future research:

- limits to usage behaviours that drive Internet infrastructure growth

- Internet disconnection as a means for promoting slow values

- Internet non-use

- the undesign of Internet services

\section{Background}

The Internet is known as a medium for acquiring and distributing information, and it can facilitate environmental benefits in some domains. For example, the total energy required for data transmission $(43 \mathrm{kWh})$ and terminal equipment $(108 \mathrm{kWh})$ for a virtualised conference was approximately 65 times lower than the energy cost for a single attendee's intercontinental round trip $(9880 \mathrm{kWh})$ [7]. Despite such benefits of the Internet, the global electricity demand for digital devices and infrastructures is rising at a rate of $7 \%$ per year - more than double the $3 \%$ annual growth of global electricity demand [37]. Preist et al. [25] have observed that demand for new digital infrastructure leads to 1) the production of new infrastructure, which 2) enables new services and 3) subsequently furthers demand. This iterative cycle emphasizes the spiraling problem of an ever-growing 'technomass' [13] of digital devices and infrastructures. Further on, as resource scarcity threatens our current levels of Internet consumption, Pargman and Wallsten 
[21] have highlighted that computing innovators should focus on providing a 'sufficient' level of service for the greatest number of people, the longest duration, and the lowest cost possible. Due to similar concerns of the Internet in a post-peak world, Raghavan and Ma [27] pose a number of questions about networking, e.g. 'Which services provided by the Internet are the least essential to its continued value and operation? Which are the most essential?'.

Previous work has investigated the energy impact of Internet demand from mobile devices, suggesting areas where Human-Computer Interaction (HCI) can be adapted for reducing such demand $[16,40]$. However, this does not engage with the subjective aspects of limiting Internet use, such as self-limitation and personal balance. This lack of subjectiveness is also present within other studies investigating the effects of Internet detoxes and forced disruptions from online media [15,33]. Whilst Kaun et al. [15] found that emotional effects (e.g. loneliness) occur due to refraining from media, their study involved asking participants to completely refrain from media for a week; this complete disconnection from the Internet could be argued more or less impossible with the way connectivity is integrated into everyday activities (especially for work). With this in mind, we aim to provide a starting point for understanding what Internet use is subjectively experienced as necessary by users.

While it is not particularly hard to realise intellectually that we ought to decrease consumption in general, as well as our consumption of digital products and services (as per [21,25,27]), it can be considerably harder to understand and accept what that entails in practice. Randall [28, p. 120] writes that:

'Changes are not necessarily easy, welcome, or attractive. They are likely to be experienced as attacks on the aspects of life that people hold dear [...] Take for example a young woman whose car is her cocoon. She has chosen it for its color and style. She fills it with personal comforts - her CDs, a favorite rug, a mascot, water-bottle, and tissues within easy reach, radio tuned to her favorite station. Snug inside, she feels safe. [...] Its outward gleam and shine speak of her success. Its inner warmth and comfort acknowledge her fragility. It both protects and expresses her identity. The suggestion that she might take the bus to work or lift-share with colleagues will not be appealing. Aspiration, lifestyle, security, and identity are all instantly under threat. We should not be surprised at the negative response to the suggestion.'

While this example is centered on car ownership and use, it forcefully conveys the affective dimensions of ownership and consumption and the emotional dimensions to such issues. The quote comes from an article that was published the better part of a decade ago, at a time when the modern smartphone was still a novelty. It is an open question if people today would be even less prone to part with their Internet-connected smartphone today than with their cars a decade ago. We are here interested in the subjective as well as objective aspects of decreased Internet access and we take an active interest of both in our exploratory study of reduced connectivity. We would also like to emphasize that the purpose of our study is neither to (quantitatively) prove something nor to in depth (qualitatively) describe and analyze some phenomenon. Rather, we aim to explore what we believe is an important phenomenon and inspire further discussions and studies in the area. While to some extent we do provide some practical suggestion in the discussion section (below), we do not feel any specific responsibility to provide a solution to the problem(s) we describe in the paper, but we wholeheartedly encourage others to do so.

\section{Experiment Design and Participants}

In order to investigate the effects of Internet disconnection, we designed a two-part, exploratory study ethically approved by the Faculty of Science and Technology Ethics department at Lancaster University, UK. The study consisted of a 'connected phase' and a 'disconnected phase' 


\begin{tabular}{|l|l|l|l|}
\hline $\begin{array}{l}\text { Participant } \\
\text { Gender, } \\
\text { Age Bracket) }\end{array}$ & Occupation & Living and Family Situation & $\begin{array}{l}\text { No. of Surveys: } \\
\text { Connected, } \\
\text { Disconnected }\end{array}$ \\
\hline P1 (F, 20s) & PhD Student & House with boyfriend & 14,14 \\
\hline P2 (F, 20s) & PhD Student & Flat with friend & 12,14 \\
\hline P3 (F, 20s) & PhD Student & Apartment with partner & 8,8 \\
\hline P4 (M, 40s) & Associate Professor & Apartment with wife and 2 children & 11,11 \\
\hline P5 (F, 20s) & PhD Student & Flat with 3 friends & 14,12 \\
\hline P6 (F, 30s) & Assistant Professor & $\begin{array}{l}\text { Apartment alone and boyfriend's } \\
\text { house with son }\end{array}$ & 14,14 \\
\hline P7 (M, 40s) & Senior Lecturer & House with partner and child & 14,14 \\
\hline P8 (F, 30s) & $\begin{array}{l}\text { Research Centre } \\
\text { Administrator }\end{array}$ & House with partner and child & 13,13 \\
\hline P9 (F, 20s) & $\begin{array}{l}\text { PhD Student with } \\
\text { part-time work }\end{array}$ & $\begin{array}{l}\text { Student accommodation with } \\
\text { partner }\end{array}$ & 16,12 \\
\hline P10 (F, 30s) & PhD Student & Apartment with partner & 12,9 \\
\hline
\end{tabular}

Table 1: An overview of the ten participants. Please note that P9 entered 16 responses for her connected phase.

lasting two weeks each. The connected phase was designed to uncover aspects of the mundane, 'baseline' use of the Internet in everyday life; participants were asked to use the Internet as they normally would and answer a 'connected phase survey' each day. Questions in this phase surrounded what the participants' days had entailed and how they had used the Internet (alone or with others), alongside reflective questions surrounding their mood.

For the disconnected phase, participants were asked to turn off Wi-Fi and/or mobile data by default on each of their Internet-enabled devices, and only turn it back on when they thought their desired Internet use was necessary. This phase was not designed to be a total Internet-free detox, but rather to treat Internet connectivity more as a need-based entity than a want-based entity; this allowed each individual to decide if, or to what extent, they could cut Internet use from their work and personal lives and how their connected lives were affected by self-imposed Internet restrictions. Like the connected phase, participants were asked to fill in a daily survey. However, this 'disconnected phase survey' included questions to capture the impacts of disconnectedness, such as: when and why the participant had turned connectivity on, whether they had re-thought the necessity to use the Internet or refrained from doing so, and further reflective questions on their mood.

The experiment ended with gathering demographic data and participants' impressions about the study overall via another survey. All three surveys consisted in a form of questions for the participants to fill in and were created on Qualtrics; these were accessed online, meaning participants had to connect to the Internet to fill them in - an obvious contradiction to our study. Having said this, one participant did fill in the daily disconnect survey offline throughout the period by downloading the questions and taking notes on a separate medium. Offline methods for gathering qualitative information would need be considered in future Internet-disconnection studies.

Ten participants took part in the study in the period of August-October 2017. These included four authors of this paper and six 'external' participants; these 'externals' were recruited through snowballing, i.e. participants personally asked others to take part. The participants are summarised in table 1 . The small sample size is heavily skewed towards academics. We find this to be less of a problem as the experiment is designed to be an initial exploratory study. The 
purpose of the study is to discover what happens when 'connectivity-off' is the default action; what we can change surrounding Internet connectivity is out of scope and inappropriate given our participants sample. Therefore we do not attempt to answer questions arising from the study, but rather inspire academics in the topic and focus future research.

For the ten participants, 128 responses were collected for the connected survey and 121 responses were collected for the disconnected survey. Whilst 140 should be expected for both phases, some participants forgot to fill out the survey every day. All of these survey responses were analysed in detail by members of the research team through coding for themes, and additional details from the authors' study participation were also added during the writing of this paper.

\section{The Connected Phase: An Overview}

It comes as no surprise that the Internet was heavily involved in the participants' working lives. Email was used regularly by all of the participants for work communication. Collaborative work was often Internet-connected through the use of cloud services such as Google Docs for paper writing, document sharing and backups (P1, P2, P3, P4, P5, P6, P9, P10) and via virtual meeting facilities such as Skype (P1, P3, P4, P6, P7, P9, P10). The participants' occupations often involved searching for information online (P6, P7, P8, P9), or browsing for online articles and literature downloads (P2, P3, P4, P5, P9, P10); teaching-related jobs further required Internet use by accessing online learning environments (P4, P6) and incorporating YouTube videos $(\mathrm{P} 4, \mathrm{P} 6)$ or podcasts $(\mathrm{P} 4)$ within academic classes.

All participants used the Internet for entertainment. This included watching on-demand content (P1, P3, P5, P7, P8, P9, P10); listening to online podcasts (P3, P4, P10), streaming music (P1, P2, P3, P4, P5, P10) or radio (P7, P8); playing Internet-connected games (P4, P7); and visiting a hobby site (P6). Entertainment practices were often shared with other people: watching YouTube videos with friends (P1, P10) and streaming Netflix or Amazon content with partners or children (P1, P3, P7, P8, P9, P10); listening to Spotify with household members (P1, P8); and gaming online with friends abroad (P7).

Interaction with other people was facilitated by Internet-connected means of communication, with instant messaging services such as WhatsApp and Facebook Messenger enabling the participants to connect with friends and family (all participants except P4); this was particularly important for overseas communication (P1, P2, P7, P8). Video-chat services (e.g. FaceTime) or social networks (e.g. Instagram) similarly enabled keeping in touch within their daily lives; only two of the ten participants did not partake in such social services during the connected phase $(\mathrm{P} 1, \mathrm{P} 7)$.

Other common Internet activities concerned online shopping or browsing to buy (all participants except P2), keeping up-to-date on the news or weather (P7, P8, P10), using financialrelated services (P1, P6, P9, P10), searching for particular information such as recipes or reviews (P1, P2, P3, P6, P7, P8, P9, P10), and for travel or navigation purposes (P1, P3, P4, P5, P6, P7, P8, P10). More irregular activities included downloading printer drivers (P4), spreading political propaganda (P3), advertising Yoga classes (P6), and child-related duties like baby tracking (P7, P8) or online homework help (P4). The survey responses emphasize that the Internet is seamlessly integrated into many aspects of the participants' lives, and whilst there are common habits and Internet dependencies, the levels of Internet usage varies among the participants. 


\section{The Disconnected Phase: The Findings}

During the disconnected phase, all the participants developed different types of workarounds to cope with their everyday practices with or without the Internet. These ways of adapting highlight the differing necessities of the participants' Internet-connected practices: the more necessary the action, the more likely the participant would connect to the Internet. We have discovered five themes relating to how participants used (or did not use) the Internet, which we have listed in order of increasing 'Internet necessity': 1) removing use completely; 2) replacing use with other activities; 3 ) planning for limited use (including pre-disconnection reliability); 4) rationing, postponing and batching use; and 5) abandoning disconnection. These are elaborated below.

\subsection{Removing Internet use completely}

All of the participants discovered some Internet connectivity that they did not find necessary, and as a result, they were able to remove such use completely from their disconnected phase. These instances were often associated with entertainment: streaming music at home (P1, P2, $\mathrm{P} 4)$; streaming video before bed or during work tasks (P3, P5); listening to podcasts whilst exercising or commuting (P3, P10); playing Pokémon Go alone (P4); and accessing favourite websites (e.g. Quora for P2) or social networks (P1, P2, P3, P10). Online searches or checks were also removed from everyday life. To name a few: P1 did not check the opening times for a shop before going to it (with the risk of it being closed); P2 had uninstalled Instagram on phone to refrain from checking updates; P5 thought it was 'fine to be offline for a while' during a train journey and whilst at a conference; P6 refrained from searching online for something personal as she reflected on previous similar searches and realised it wouldn't be helpful; P7 wanted to search for the range of a MW radio or the actors, directors, and writers of a film yet found it easy to refrain; P8 didn't search for a post office nearby when her usual one was closed (instead just went to another she knew of); and P9 didn't check her emails during a lunch break at a training course. We name this category the most unnecessary Internet use since participants were able to easily avoid such online activities in their everyday life.

\subsection{Replacing Internet use with other activities}

All of the participants (except P4) were able to find some other activity to replace part of their previous Internet-connected activities. For communicating, instant messaging services were replaced by SMS for 5 of the 10 participants (P1, P3, P6, P7, P8). P2 even replaced her Wi-Fi calls with cellular, despite the extra charges for contacting friends and family overseas; this was similar to P7 in some instances when contacting his friend abroad.

On-demand content was also frequently replaced; rather than streaming or downloading, participants relied on local or broadcast content such as DVDs (P1, P7, P8), broadcast TV $(\mathrm{P} 1, \mathrm{P} 5, \mathrm{P} 9, \mathrm{P} 10)$, the radio $(\mathrm{P} 1, \mathrm{P} 3, \mathrm{P} 5, \mathrm{P} 10)$ and stored music $(\mathrm{P} 1, \mathrm{P} 3, \mathrm{P} 5)$. This sometimes caused major changes in participants' activities. For example, P10 and her partner decided to go to the cinema one evening instead of streaming something on Netflix, and P1 fixed a problem with her broadcast television to ensure she could watch the Great British Bake Off live. P7 got up early one morning to locate his old 'family heirloom' radio to facilitate his listening practice; this led him to listen to a different radio station entirely due to his usual 'BBC World News' no longer broadcasting on shortwave radio in Europe.

Participants sometimes substituted the time that they would normally use for Internet entertainment with completely different activities. Some examples include: reading (P1, P2, 
P3, P5, P8, P9, P10); household activities such as cooking or cleaning (P5, P10); creative tasks e.g. writing and photo development (P3, P5); playing card games and offline PC games (P3); and even activities for the self, such as sleeping, bathing and meditating (P1, P2, P8). In some cases, evenings at home with a partner that would usually involve watching on-demand content instead resulted in chatting and spending time together (P1, P3). We name these replaced activities as the second-most unnecessary Internet-involved tasks, rather than the first-most, due to the fact that participants noted replacing their use of the Internet with something else.

\subsection{Planning for limited Internet use and pre-disconnection reliability}

Some forms of planning for 'being offline' were developed by the participants in order to carry out disconnection without majorly affecting themselves or others. To avoid missing her favourite TV shows during the study, P1 recorded programmes on her parents' Sky+ box as she doesn't have a set-top box at home. Extra planning was also required by the participants who cycle to work (P7, P10), as they did not deem weather forecasting checks via the Internet necessary at the beginning of the study. This meant that rain gear was packed regardless of the weather forecast. However, once P10 had taken her rain coat to work on a day which turned out to be sunny, she figured out when the weather forecast was broadcasted on television each morning and watched that in order to plan her outfit and avoid carrying her raincoat unnecessarily again.

Furthermore, participants sometimes relied on connectivity during the connected phase, or within brief connected periods in the disconnected phase, to prepare for Internet disconnectivity. Participants pre-downloaded music or podcasts (P1, P3, P10) and watched pre-downloaded content (P1, P3). This reliance was also needed to avoid missing out on messages from others: P7 asked his friend overseas to contact him via SMS (despite the extra costs) and P1 forewarned friends and family to contact her via SMS or phone call if they needed her immediately. This warning activity of slow responses was similar to that of other participants: P3 posted updates on her Instagram and blog that she would not be posting for the coming weeks, P5 changed her WhatsApp profile picture to 'offline' when she wasn't checking it, and both P3 and P6 added that they would be checking emails less often to their work email reply footers. As Internet connectivity is sometimes required for, or integrated in, planning for periods of disconnection, we argue that this connectivity category is next in the order of increased necessity.

\subsection{Rationing, postponing and batching Internet use}

For some activities, Internet use was required and could not be removed from everyday life, replaced with disconnected tasks, or planned for via other means. Despite this, participants were able to ration their use of the Internet for activities or postpone them to times of connectivity, leading to batched uses of the Internet. For rationing use, participants limited connectivity: when family members were absent (P4); via device (P3) or location (P5); or by the number of connectivity instances. The latter here included checking emails only twice or thrice daily (P1, P5), checking instant messaging networks once a day (P1) or only during breaks (P5), limiting podcast listening to only three (time-sensitive) podcasts per week (P4), and refreshing news and weather apps each evening (P7). With rationing, participants declare what is their minimum level of connectivity required for maintaining their everyday practices.

Postponing, however, highlights how connectivity is required for the task - yet instant access to the Internet is not necessary. These types of activities were delayed to times where other activities required connectivity, for example P1 only did things online during her thrice-daily email checks whereas P7 would put off his email checks until he had to connect to the Internet 
for other means. This was similar to P10, who reflected that she would put off online tasks but knew she would have to do them at some point anyway. Other in-study postponed activities included searching for addresses (P6) and replying to emails or messages (P5, P9), yet some practices such as watching the rest of a streamed film (P7) were postponed until after the study entirely. We rank this as the second-most necessary action since Internet use was a requirement for the reduced or delayed tasks to be carried out.

\subsection{Abandoning disconnection completely}

It was obvious from the initial discussion about the study that complete Internet disconnection was going to be impossible when the Internet is so tightly integrated into the many things that we do in everyday life; this was evident within the disconnected phase, particularly for the participants' work practices. Whilst two participants did find ways to work offline through using books (P1) or searching for emails via Thunderbird's offline mode (P7), participants heavily suggested that they cannot, or find it very difficult to, fully disconnect during their work day. P4 mentioned in frustration that he 'just * can't* disconnect and still get work done. Writing a paper $=$ using Google docs for starters'; this Internet use for collaborative writing was common amongst many participants (P2, P3, P4, P6, P7, P10). Internet-connectivity seeped into other work situations: uploading or retrieving information from virtual learning environments (P6, P7); researching literature or articles (P2, P3, P5, P9, P10); and (most frequently) for email, a connectivity necessity for all participants. Less common reconnection practices included P8's requirement to check her workplace's Twitter - her slight differentiation in job role shows that other work styles can still necessitate Internet-connectivity. Despite Internet-free working days being classified as impossible for the participants, they did reflect that disconnecting from the Internet proved helpful for their levels of productivity, focus and face-to-face interaction at work and within meetings (P1, P2, P3, P4, P5, P6, P10); this was achieved through limiting email (P1, P5, P10), reduced Internet procrastination (P2, P3, P5, P9), and by the 'opportunity to think and talk instead of reduced-attention-surfing' (P4).

The study also revealed non-work related situations in which participants abandoned disconnection. Reasons for connecting surrounded: participants' stresses and counteracting bad moods (P3, P10), contacting friends (P6, P7), accessing on-demand content to a watch film with family (P1, P10), and accidental use of the Internet through what was thought to be an offline game (P1). Participants commonly returned to connectivity for travel or navigation requirements: P1 needed to use Google to find an address; P5 needed to use Google Maps at a conference for directions (despite asking locals first) and P10 needed to access Google Maps when running late to a meal in order to find the fastest way there. Unless not turning up at their desired location was an option, it can be argued that P1, P5 and P10 had 'no choice' in these situations. This lack of choice in Internet use for this category is why we deem it as the most necessary form of use.

\section{Discussion}

From our findings of Internet-disconnection adaptation, we are able to suggest four areas of future research: 1) for creating limits to usage behaviours that drive Internet infrastructure growth; 2) for the promotion of slow values through Internet disconnection; 3) for Internet non-use; and 4) for discussing the undesign of Internet services. We examine each of these below. 


\subsection{Limits to behaviours that drive Internet infrastructure growth}

Real-time (i.e. on-demand) entertainment contributed to the largest portion of peak-period traffic in 2016 for North America [31] and in 2015 for Europe [30]; Preist et al. [25] states this connected entertainment as a 'significant driver' in the expansion of Internet infrastructure. Our survey responses showed, however, that on-demand content such as streaming online music or video was repeatedly removed or replaced by other non-connected activities in the disconnected phase.

Due to the way the Internet is currently manifested into work roles and other activities, our survey data backs up the fact that a 'digital day of rest' for reduced global energy use [6] would be near impossible for today's society unless it is restricted only to leisure (not encompassing work). Despite this, days of reduced connectivity for necessary-only online actions are not as intolerable. People adapt to Internet disconnection, particularly in areas that drive infrastructure growth. We therefore argue, not just that there should be limits to the Internet usage behaviours that drive infrastructure growth, but rather that there can be such limits. We propose that exploring these limits to Internet connectivity is an important and valid area for future research. As a cautionary note however, we believe this future work should take into account rebound effects; whilst this was not the focus of our study, the user practices resulting from adapting to Internet disconnection could potentially be less sustainable and therefore it is worth considering whether each proposed limit to Internet connectivity is worthwhile.

\subsection{Slow values}

Slow food (The Slow Food Manifesto $1989^{1}$ ) and the more general slow movement ${ }^{2}$ have inspired many offshoots, e.g. slow education [1], slow scholarship ${ }^{3}$ [5], slow technology $[11,22,26]$ and slow design $[9,35]$. It is also possible to relate slowness and the slow movement to other phenomena such as the transition movement [12], the degrowth movement [8], simple living [10], downshifting [34], off-the-grid living [38], the tiny house movement etc. From these inspiring slow-movement based perspectives, the major take-away is that their respective approaches all support a slowdown of, and, by extension, a reversal of Internet infrastructure growth.

Although most working day practices from our analysis involve the Internet, our participants experienced improved focus and productivity during their disconnected phase. Previous work has shown that working without email can improve task concentration [19] and reduce stress [17], and we extend this to Internet disconnectivity in general: Internet-connected tasks can be batched and postponed; online procrastination habits broken; people are able to focus on one thing at a time. This increased concentration shows how Internet-disconnection is a mechanism for promoting values of slow technology design, i.e. through encouraging 'mental rest' and 'slower, less consumptive lifestyles and practices' [20]. Therefore, we emphasise that reduced Internet connectivity should be exploited for slowing the pace of life, particularly since ICT can lead to a 'more densely packed everyday life' and therefore intensify the energy associated with day-to-day practices [29].

\subsection{Internet non-use}

Research in HCI has explored varieties of technology non-use [32], dimensions of non-users [41] and issues of non-use [2-4]. In an initial study of technology non-use, Baumer et al. [2] describe

\footnotetext{
${ }^{1}$ https://goo.gl/J8jpqW and https://www.slowfood.com

2 https://en.wikipedia.org/wiki/Slow_movement_(culture)

${ }^{3}$ http://web.uvic.ca/ hist66/slowScholarship/
} 
various non-use Facebook behaviors. More specifically: 1) practices of not using Facebook in the first place; 2) practices surrounding leaving Facebook (permanently by deleting or deactivating an account or quitting whilst leaving the account activated) and relapsing (leaving and later returning to Facebook); and 3) practices of limiting Facebook use. Baumer et al. [2] also discuss the effects of leaving or limiting Facebook use in terms of reactions from Facebook friends 'left behind'. Despite these practices being one aspect of Internet non-use and disconnection, our study is not limited to social media and focuses on broader Internet use.

There are naturally several parallels between our finding 'Removing Internet use completely' and the Baumer et al. [2] practice of 'relapsing'. Based on our post-study survey, many participants who removed certain kinds of Internet use (e.g. streaming music or video, playing Pokémon Go or accessing social networks) noted that they had mostly returned to their prestudy behaviors ('relapsed'). Some participants also mentioned that the 'Internet diet' led them to decrease Internet use in particular activities after the study ended but we do not know whether modes of quitting or limiting Internet use are or will be more permanently sustained. There are further parallels between our finding 'Rationing, postponing and batching Internet use' and the Baumer et al. [2] theme of 'limiting'. However, the examples that Baumer et al. [2] show are more 'severe' than the practices observed here (e.g. writing a dissertation and '[having] my husband change my password and he'd log me on once a week or so as a treat').

Whilst Baumer et al. [2] provide four motivation practices for Facebook non-use (privacy, data use and misuse, banality and productivity), we propose these can be extrapolated to Internet non-use in general with the additional motivation of sustainability. Although it is hard to imagine that users would restrict their Internet use for sustainability, it is considerably easier to imagine that these motivations can be combined, e.g. restricting Internet use due to reasons pertaining to sustainability and data misuse and productivity. 'Assembling' combinations of various motivations would overcome the hard-to-sell nature of sustainability whilst making it more successful and constitutes a powerful reason for further exploring Internet non-use.

\subsection{The undesign of Internet services}

As with technology non-use, HCI research has also explored issues of undesigning technology [23, 24]. Pierce [23] more theoretically discusses 'undesign', or, 'the intentional negation of technology' such as designs that 'hinder or prevent the use of technology in particular ways and contexts' [23]. This is the opposite of how most Internet services are currently designed: Netflix makes it easier to binge watch TV shows by cutting out the credits and automatically playing the next episode; Facebook tries to make users log back on through pushing notifications and making them worry about missing out if they don't repeatedly check what has happened in the last day, hour or minute.

It should be noted that Pierce primarily discusses the design of technology while we limit our discussion to restrictions in our behaviour and in our use of technology. While informants in our study have practiced 'self-inhibition' in their daily lives, Pierce [23] uses the same term ('selfinhibition') to refer to 'the design of attributes of a technology that inhibit use of that technology in various ways'. While Pierce's terms are relevant to us, there are important differences in terms of where responsibility is located; we place responsibility in the individual whereas Pierce places it in the (design of the) technology itself, and by extension in designers rather than users of (digital) technologies. A particular case of self-inhibition, 'self-inhibiting options' [23] comes the closest to bridging this gap, since such options refer to the design of settings that the individual user can configure to inhibit or prevent certain uses of the technology (e.g. parental controls). Pierce also notes that while designers often design settings that users can configure, 
they seldom design 'options that encourage or allow individuals to reflectively and explicitly self-inhibit their own use' - the exact kind of support that our study participants would have wanted.

In our experience, most participants changed the default setting on their devices to 'offline' and 'logged out' for the study duration, choosing only to connect to the Internet when they determined it was necessary. This is an example of self-inhibition as well as of designers choosing to make such settings available to end users. Yet simply turning Internet connectivity on or off can be difficult for some devices (as found with P6) and often means removing connectivity for all services or none at all (as noted by P1). In fact, operating systems and applications could (and can) easily require constant connectivity to work in their totality. While it wasn't that many years ago since you got logged out from many services due to inactivity, it is now possible to be connected to Google, to Facebook and to many other services for days or weeks on end, i.e. 'forever'. Based on our study and through taking undesign goals and principles into consideration, we argue that it should be made easier for users to disconnect from the Internet and specific services accessed through it. For example, through returning automatic service logouts after a period of inactivity, or even through easier, service-orientated connectivity management.

\section{Conclusion}

In this study, we have explored participants' subjective limits to reduced Internet connectivity. Participants were able to define their 'necessary' Internet use through batching, rationing and planning for (or abandoning) disconnection. For more 'unnecessary' uses of the Internet, participants were often able to refrain from particular online activities (e.g. streaming on-demand content or accessing social media) or found other means to replace them. Internet use optimisation proved to be possible without compromising subjective personal, professional and social needs, and we have shown that reduced Internet connectivity is relevant for a number of research areas both within and outside of sustainability. With this as a standpoint, we urge the research community to further investigate reduced Internet use. Future work would greatly benefit the topic by investigating Internet disconnection on a larger scale, for different user demographics, for groups outside of everyday users (e.g. businesses), or through deeper ethnographic studies to uncover nuances in long-term actions from Internet disconnection and the costs of it (e.g. inconvenience or missed opportunities).

Whether it is a motivation or not within future work, sustainability as an outcome of reduced Internet connectivity is certainly a potential direction for a sustainable future. Yet, it could be argued that the problem (excessive Internet use etc.) should not focus on individuals' everyday Internet use but rather at some other level, for example in terms of policy. It is certainly true that many proposals (for reducing GHG emissions etc.) firmly place the burden of 'saving the world' on the shoulders of individuals rather than on more powerful stakeholders. We however believe that reducing Internet connectivity is just as much a matter of 'inner change', of reflecting upon and curtailing 'needless' Internet use as part of practicing 'temperance', of attaining peace of mind and in terms of personal development. Do we really need to be always connected and what do we lose when we can't put our smartphones down? Our suggestions concerning the redesign of affordances of various tech companies' (Facebook, Netflix) services are situated at a level in between the individual and regulation but it might be the case that change at that level will only happen through regulation. 


\section{Acknowledgements}

We would like to thank the six external participants for their cooperation and involvement within this study. We thank our reviewers, Maria Håkansson, and other academics that have provided us with valuable feedback and discussion, particularly in regards to outlining future work. We would also like to thank the participants and organisers of the ICT4S Summer School 2017 and the Lorentz Center in Leiden, both for introducing us as co-authors and motivating us to carry out this research. We further acknowledge the EPSRC (grant number: W95738G), for without whom this work would not be possible. As agreed with the participants in our experiment, and to maintain both privacy and external participant confidentiality, we have not made the survey data publicly available.

\section{References}

[1] Irena Barker. Find the time for slow education. Times Educational Supplement Scotland, 2290:26, 2012.

[2] Eric PS Baumer, Phil Adams, Vera D Khovanskaya, Tony C Liao, Madeline E Smith, Victoria Schwanda Sosik, and Kaiton Williams. Limiting, leaving, and (re) lapsing: an exploration of facebook non-use practices and experiences. In Proceedings of the SIGCHI conference on human factors in computing systems, pages 3257-3266. ACM, 2013.

[3] Eric PS Baumer, Morgan G Ames, Jenna Burrell, Jed R Brubaker, and Paul Dourish. Why study technology non-use? First Monday, 20(11), 2015.

[4] Eric PS Baumer, Jenna Burrell, Morgan G Ames, Jed R Brubaker, and Paul Dourish. On the importance and implications of studying technology non-use. interactions, 22(2):52-56, 2015.

[5] Maggie Berg and Barbara Seeber. The slow professor: Challenging the culture of speed in the academy. University of Toronto Press, 2016.

[6] Eli Blevis, Chris Preist, Daniel Schien, and Priscilla Ho. Further connecting sustainable interaction design with sustainable digital infrastructure design. In Proceedings of the 2017 Workshop on Computing Within Limits, pages 71-83. ACM, 2017.

[7] Vlad C Coroama, Lorenz M Hilty, Ernst Heiri, and Frank M Horn. The direct energy demand of internet data flows. Journal of Industrial Ecology, 17(5):680-688, 2013.

[8] G D'Alisa, F Demaria, and G Kallis, editors. Degrowth: a vocabulary for a new era. Routledge, 2014.

[9] Barbara Grosse-Hering, Jon Mason, Dzmitry Aliakseyeu, Conny Bakker, and Pieter Desmet. Slow design for meaningful interactions. In Proceedings of the SIGCHI Conference on Human Factors in Computing Systems, pages 3431-3440. ACM, 2013.

[10] Maria Håkansson and Phoebe Sengers. Beyond being green: simple living families and ict. In Proceedings of the SIGCHI Conference on Human Factors in Computing Systems, pages 27252734. ACM, 2013.

[11] Lars Hallnäs and Johan Redström. Slow technology-designing for reflection. Personal and ubiquitous computing, 5(3):201-212, 2001.

[12] Rob Hopkins et al. The transition handbook. From oil dependency to local resilience, 2008.

[13] Alf Hornborg. The power of the machine: Global inequalities of economy, technology, and environment. Rowman Altamira, 2001.

[14] ITU. Ict facts and figures 2017. Technical report, ITU, 2017. http://www.itu.int/en/ITU-D/ Statistics/Documents/facts/ICTFactsFigures2017.pdf, accessed October 2017.

[15] Anne Kaun and Christian Schwarzenegger. " no media, less life?" online disconnection in mediatized worlds. First Monday, 19(11), 2014. 
[16] Carolynne Lord, Mike Hazas, Adrian K Clear, Oliver Bates, Rosalind Whittam, Janine Morley, and Adrian Friday. Demand in my pocket: mobile devices and the data connectivity marshalled in support of everyday practice. In Proceedings of the 33rd Annual ACM Conference on Human Factors in Computing Systems, pages 2729-2738. ACM, 2015.

[17] Gloria Mark, Stephen Voida, and Armand Cardello. A pace not dictated by electrons: an empirical study of work without email. In Proceedings of the SIGCHI Conference on Human Factors in Computing Systems, pages 555-564. ACM, 2012.

[18] Susan Maushart. The winter of our disconnect. ReadHowYouWant. com, 2011.

[19] Cal Newport. Deep work: Rules for focused success in a distracted world. Hachette UK, 2016.

[20] William Odom, Richard Banks, Abigail Durrant, David Kirk, and James Pierce. Slow technology: critical reflection and future directions. In Proceedings of the Designing Interactive Systems Conference, pages 816-817. ACM, 2012.

[21] Daniel Pargman and Björn Wallsten. Resource scarcity and socially just internet access over time and space. In Proceedings of the 2017 Workshop on Computing Within Limits, pages 29-36. ACM, 2017.

[22] Norberto Patrignani and Diane Whitehouse. Slow tech: a quest for good, clean and fair ICT. Journal of Information, Communication and Ethics in Society, 12(2):78-92, 2014.

[23] James Pierce. Undesigning technology: considering the negation of design by design. In Proceedings of the SIGCHI Conference on Human Factors in Computing Systems, pages 957-966. ACM, 2012.

[24] James Pierce. Undesigning interaction. interactions, 21(4):36-39, 2014.

[25] Chris Preist, Daniel Schien, and Eli Blevis. Understanding and mitigating the effects of device and cloud service design decisions on the environmental footprint of digital infrastructure. In Proceedings of the 2016 CHI Conference on Human Factors in Computing Systems, pages 13241337. ACM, 2016.

[26] Andrew Price. Slow-tech: Manifesto for an over-wound world. Atlantic Books Ltd, 2009.

[27] Barath Raghavan and Justin Ma. Networking in the long emergency. In Proceedings of the 2nd ACM SIGCOMM workshop on Green networking, pages 37-42. ACM, 2011.

[28] Rosemary Randall. Loss and climate change: The cost of parallel narratives. Ecopsychology, 1(3):118-129, 2009.

[29] Inge Røpke and Toke Haunstrup Christensen. Energy impacts of ict-insights from an everyday life perspective. Telematics and Informatics, 29(4):348-361, 2012.

[30] Sandvine. Global internet phenomena report. asia-pacific and europe. Technical report, Sandvine Incorporated ULC, 2015.

[31] Sandvine. Global internet phenomena report. latin america and north america. Technical report, Sandvine Incorporated ULC, 2016.

[32] Christine Satchell and Paul Dourish. Beyond the user: use and non-use in HCI. In Proceedings of the 21st Annual Conference of the Australian Computer-Human Interaction Special Interest Group: Design: Open 24/7, pages 9-16. ACM, 2009.

[33] Sarita Yardi Schoenebeck. Giving up twitter for lent: how and why we take breaks from social media. In Proceedings of the SIGCHI Conference on Human Factors in Computing Systems, pages 773-782. ACM, 2014.

[34] Juliet Schor and Karen Elizabeth White. Plenitude: The new economics of true wealth. Penguin Press New York, 2010.

[35] Carolyn Strauss and Alastair Fuad-Luke. The slow design principles. Proceedings of the Changing the Change, 2008.

[36] Sherry Turkle. Alone together: Why we expect more from technology and less from each other. Basic books, 2011.

[37] Ward Van Heddeghem, Sofie Lambert, Bart Lannoo, Didier Colle, Mario Pickavet, and Piet Demeester. Trends in worldwide ict electricity consumption from 2007 to 2012. Computer Commu- 
nications, 50:64-76, 2014.

[38] Phillip Vannini and Jonathan Taggart. Off the grid: re-assembling domestic life. Routledge, 2014.

[39] Judy Wajcman and Emily Rose. Constant connectivity: Rethinking interruptions at work. Organization Studies, 32(7):941-961, 2011.

[40] Kelly Widdicks, Oliver Bates, Mike Hazas, Adrian Friday, and Alastair R Beresford. Demand around the clock: time use and data demand of mobile devices in everyday life. In Proceedings of the 2017 CHI Conference on Human Factors in Computing Systems, pages 5361-5372. ACM, 2017.

[41] Sally ME Wyatt, N Oudshoorn, and T Pinch. Non-users also matter: The construction of users and non-users of the internet. Now Users Matter: The Co-construction of Users and Technology, pages $67-79,2003$. 